\title{
Safety profile of chloroquine and hydroxychloroquine: an analysis of the FDA Adverse Event Reporting System (FAERS) database
}

\author{
Georgios Papazisis $^{1}$, Spyridon Siafis ${ }^{1}$, Dainora Cepaityte $^{1}$, Dimitrios Giannis ${ }^{2}$, Dimitrios \\ Tzachanis $^{3}$, and Antoine Egberts ${ }^{4}$ \\ ${ }^{1}$ Aristotle University of Thessaloniki Faculty of Health Sciences \\ ${ }^{2}$ Northwell Health Feinstein Institutes for Medical Research \\ ${ }^{3}$ University of California San Diego School of Medicine \\ ${ }^{4} \mathrm{UMC}$ Utrecht
}

May 19, 2020

\begin{abstract}
Aim: CQ and HCQ are currently being investigated worldwide for their clinical efficacy against COVID-19, however a major concern regarding these drugs remains their safety profile. The aim of the present study was to identify potential safety signals of $\mathrm{CQ}$ and $\mathrm{HCQ}$ use, in the period prior to their repurpose as COVID-19 treatment options analyzing safety data retrieved from the FDA Adverse Event Reporting System (FAERS) pharmacovigilance database. Methods: We performed a disproportionality analysis of all available FAERS data between the first quarter of 2004 and December 2019 using the OpenVigil2.1-MedDRA software. Disproportionality was quantified using the reporting odds ratio (ROR) and its $95 \%$ confidence interval (CIs). The reporting mortality of CQ and HCQ was also investigated Results: The dataset contained 6,635,356 reports. Based on the comparison of the RORs, significant differences were observed between CQ and HCQ for most of the adverse events: cardiomyopathy, cardiac arrhythmias, retinal disorders, corneal disorders, hearing disorders, headache, hepatic disorders, severe cutaneous reactions, musculoskeletal disorders and cytopenia. Only CQ was significantly associated with psychotic disorders, suicide and self-injury, convulsions, peripheral neuropathy and decreased appetite. In multivariable logistic regression, outcome death was more frequently associated with CQ users, generally older females, with co-reported suicide and self-injury, cardiomyopathy, cardiac arrhythmias and decreased appetite. Discussion: Our results suggest that HCQ has a safer clinical profile compared to CQ, especially regarding cardiotoxicity and thus could serve as a safer therapeutic approach in COVID-19. However, until more real-world and RCTs' data are available, close supervision is strongly recommended.
\end{abstract}

\section{Introduction}

In the era of the COVID-19 pandemic, there is an urgent need to find effective drugs to treat and/or prevent the disease spread. The more than 50 years old drugs Chloroquine (CQ) and its structural analog hydroxychloroquine (HCQ), have been used for decades as the primary and most successful drugs against malaria and certain autoimmune diseases. Based on their endosomal-lysosomal acidification inhibitory action and their anti-inflammatory and immunomodulatory effects, CQ and HCQ are currently being investigated for their clinical efficacy against COVID-19. Over 80 clinical trials are under way worldwide using $\mathrm{Cl}$ or HCQ in the treatment and/or prophylaxis of COVID-19 as a monotherapy or in combination with other drugs [1].

Preliminary clinical evidence supporting the efficacy of HCQ were published on March 20 by the team of D. Raoult in Marseille [2]. However, the publishing journal's society subsequently declared that the trial did "not meet the Society's expected standard'[3].Early results from a multicenter trial in China suggested also that CQ may limit exacerbation of pneumonia and shorten viral replication and course of COVID- 
19 disease [4]. On March 28th the FDA issued an Emergency Use Authorization for emergency use of chloroquine phosphate and hydroxychloroquine sulfate by healthcare providers in the treatment of COVID19 [5].However, the evidence regarding their clinical efficacy remains conflicting and a sufficiently powered clinical trial has not yet been reported. Thus, it is unclear if there are therapeutic benefits of CQ or HCQ administration to SARS-CoV-2-infected patients.

Several concerns have recently emerged, due to the severe cardiac complications potentially resulting from the use of this treatment in COVID-19 patients [6,7]. On April 23 $3^{\text {rd }}, 2020$ the European Medicines Agency (EMA) published a public health statement issued by the COVID-19 EMA pandemic Task Force (COVIDETF) in consultation with EMA's safety Committee (PRAC), in light of the ongoing discussions on the use of CQ and HCQ in the treatment of COVID-19 [8]. The statement is a reminder of the risk of serious side effects with CQ and HCQ use, based on the results of recent studies that have reported serious, in some cases fatal, arrhythmias with CQ or HCQ, particularly when these drugs are taken at high doses or in combination with the antibiotic azithromycin [9]. One day later, on April $24^{\text {th }}, 2020$ FDA released a drug safety communication recommending the use of HCQ or CQ for COVID-19 only in hospital and clinical trial settings[10].

Since CQ derivatives are under clinical trial evaluation and remain a therapeutic option for COVID-19, the availability of human safety data remains a priority to weigh the benefits to the risks. Pharmacovigilance databases, like the US Food and Drug Administration Adverse Event Reporting System (FAERS) contain valuable real-world data on suspected adverse drug reactions. Thus, the aim of the present study was to identify potential safety signals of CQ and HCQ use, in the period prior to their repurpose as COVID-19 treatment options, using a disproportionality analysis of all available FAERS data between the first quarter of 2004 and December 2019.

\section{Methods}

i. Database

The US Food and Drug Administration (FDA) Adverse Event Reporting System (FAERS) is a pharmacovigilance database including U.S. American and international data on suspected adverse drug reactions including administrative information, patient demographics, adverse events, information about drug therapy and patient outcomes [11]. OpenVigil2.1-MedDRA software is an open data-mining tool incorporating the MedDRA terminology (Introductory Guide for Standardized MedDRA Queries (SMQs) Version 22.0.; 2019) that provides the ability to access and analyze cleaned FAERS data (verified and normalized drug names). Access to individual reports as well as counts of safety reports meeting specific criteria is possible. The OpenVigil2.1-MedDRA software was used to retrieve reports submitted between the first quarter of 2004 and December 2019 (period available via OpenVigil2.1-MedDRA).

ii. Case/non-case analysis of the safety profile of chloroquine and hydroxychloroquine

First, we conducted a case/non-case analysis to investigate the safety profile of CQ and HCQ. The following list of adverse events were selected after inspection of the Summary Product Characteristics of both drugs: cardiomyopathy, cardiac arrhythmias, convulsions, headache, extrapyramidal symptoms, peripheral neuropathy, musculoskeletal disorders, depression, psychotic disorders, suicide and self-injury, corneal disorders, retinal disorders, hearing and vestibular disorders, gastrointestinal symptoms, hepatic disorders, decreased appetite, lactose intolerance, hypoglycemia, DRESS, hypersensitivity and haematopoietic cytopenia. The above adverse events were matched to MedDRA terms given priority to standardized MedDRA Queries (SMQs) and higher-level terms (Table S1). Reports associated with CQ or HCQ were used to define exposure and all other events and drugs in the database were defined as non-cases and non-exposure, respectively.

Disproportionality analysis was performed to detect a safety signal for reporting each of the investigated adverse events and either CQ or HCQ. Disproportionality was quantified using the reporting odds ratio (ROR) and its $95 \%$ confidence interval (CIs). ROR estimates the frequency of the examined adverse event 
co-reported with the drug of interest compared with all other drugs in the database. Disproportionality signals were defined when the lower boundary of the $95 \%$ CI of ROR was greater than one and the number of reports was higher than three [12]. RORs and their confidence intervals were conducted in OpenVigil2.1MedDRA.

iii. Reporting death in chloroquine and hydroxychloroquine reports

Further, we investigated the reporting mortality of CQ and HCQ. The sample consisted of reports with CQ or HCQ, which were extracted using OpenVigil2.1-MedDRA and additional duplicates were excluded. Multivariable logistic regression was conducted using as dependent variable the outcome of the report (death or not) and as independent variables the presence of one of the above MedDRA terms (including arthritis, malaria, systematic lupus), age, sex, reporting year, reporting country (US or not US) and drug (CQ or HCQ). Complete case analysis was conducted and we excluded reports with missing data concerning the age, sex, reporting year, reporting country, drug name or outcome. Alpha was set at two-sided 0.05 and analysis was performed in $\mathrm{R}$ version 3.6.1.

\section{Results}

The dataset contained 6,635,356 reports submitted in FAERS between the first quarter (Q1) of 2004 and December (Q4) 2019. CQ was identified in $942(0.014 \%)$ reports and HCQ in 25,862 (0.390\%). Disproportionality signals with the RORs are reported in Table $\mathbf{1}$ and Figure 1.Based on the signal results, both CQ and HCQ were associated with reporting cardiomyopathy, cardiac arrhythmias, retinal disorders, corneal disorders, hepatic disorders, hearing and vestibular disorders, severe cutaneous reactions, headache, musculoskeletal disorders and haematopoietic cytopenia. CQ was further significantly associated, showing RORs indicative of a safety signal, with psychotic disorders, suicide and self-injury, convulsions, peripheral neuropathy and decreased appetite, while no signal was detected for HCQ for these adverse events. HCQ was additionally associated with reporting hypersensitivity and gastrointestinal symptoms.

Based on the comparison of the RORs, significant differences can be observed between CQ and HCQ indicating a more favorable safety profile of HCQ for most of the selected adverse events: cardiomyopathy (22.2 vs 3.2 for CQ and HCQ respectively), cardiac arrhythmias (CQ: 7.6 vs HCQ: 1.2), retinal disorders (CQ:12.6 vs HCQ:4.2), corneal disorders (CQ:8.7 vs HCQ:1.8), hearing and vestibular disorders (2.4 vs 1.3), headache (1.7 vs 1.5), hepatic disorders (CQ: 3.1 vs HCQ: 1.8 ), severe cutaneous reactions (2.9 vs 2.5), musculoskeletal disorders (2.0 vs 1.5$)$ and hematopoietic cytopenia (2.0 vs 1.4 ). Furthermore, safety signals identified only for CQ were risk for reporting psychotic disorders (ROR 2.8), suicide and self-injury (ROR 4.2) peripheral neuropathy (ROR 3.3), convulsions (ROR 2.6) and decreased appetite (ROR 1.9). Safety signals found only for HCQ and not for CQ were for reporting hypersensitivity and gastrointestinal symptoms, nevertheless these RORs were relatively low (1.9 and 1.4 respectively). (Table $\mathbf{2}$ and Figure 2)

To investigate reports of death associated with CQ and HCQ use, we analyzed a final sample of 10,279 reports with these drugs(Figure S1). Among this sample CQ was reported in $537(5.2 \%)$ and HCQ in $9742(94.8 \%)$ of the reports. Outcome death was reported in $755(7.3 \%)$ of the sample. In multivariable logistic regression, reports with the outcome death were more frequently associated with CQ users, generally older, more frequently females originating from the United States with co-reported suicide and self-injury, cardiomyopathy, cardiac arrhythmias as well as decreased appetite.

Discussion CQ and HCQ are indicated and have a well-established benefit in the treatment of systemic lupus erythematosus, rheumatoid arthritis and CQ additionally for treatment and prevention of malaria. CQ analogs mediate several pharmacological in vitro actions and have become potential candidates for the treatment of several emerging viral diseases, such as COVID-19. CQ and HCQ are diprotic weak bases. They become highly concentrated in acidic cytoplasmic vesicles, such as endosomes, lysosomes, or Golgi vesicles thereby increasing their $\mathrm{pH}$ and leading to dysfunction of several enzymes, e.g. those required for proteolytic processing and post-translational modification of viral proteins. By neutrality of acidic $\mathrm{pH}$ in endosomes, CQ analogs inhibit the viral entry and replication processes into the cytoplasm of susceptible cells and thereby prevent viral infections. They also exhibit anti-inflammatory and immunomodulatory properties 
due to inhibition of several cytokine production (e.g., IFN- $\gamma$ TNF-a, neopterin) from various cells and also able to prevent the activation of macrophages [13]. Further, the drugs seem to interfere with the process of terminal glycosylation of the cellular receptor angiotensin converting enzyme 2 (ACE-2), that interacts with the SARS-CoV-2 spike protein and mediates viral entry [14]. This may negatively affect the virus-receptor binding and abrogate the infection.

Although dosing for malaria and autoimmune disorders is relative well established, the optimal dosing and duration of treatment for COVID-19 is yet unknown. The suggested dose, based on the US FDA Emergency Use Authorization recommendation for hospitalized adult patients or adolescents weighing $>50 \mathrm{~kg}$ and not enrolled in an HCQ clinical trial for, is $1000 \mathrm{mg}$ per os followed by $500 \mathrm{mg}$ per os for a total treatment duration of 4 to 7 days [5]. However, previous evidence suggests that there is a considerable inter-subject variability in the steady state blood concentrations of CQ analogs. In addition, CQ is a racemic mixture. It has been reported that the kinetic behavior of separate enantiomers differs in humans, while there are no data about the relevance of stereospecificity in the therapy of emerging viral infections. Regarding to pharmacokinetic parameter considerations, CQ has been disputed for its narrow therapeutic indexes and poor penetration in specific tissues. In contrast, HCQ has a lower level of tissue accumulation, which may explain the fact that it is associated with fewer adverse events than CQ. Indeed, only high-dose and long-term (over 5 years) intake of HCQ is likely to contribute to the development of retinopathy [15].

According to the drugs summary of product characteristics very common side effects of both drugs include abdominal pain and nausea and common side effects include anorexia, diarrhoea, vomiting, headache, skin rash, pruritus and blurring of vision (impaired accommodation). Although databases like FAERS contain usually more severe side effects, since the mildest ones are underreported, some of the common events were captured in our analysis as safety signals (decreased appetite, gastrointestinal symptoms, hypersensitivity, severe cutaneous reactions, headache). Interestingly, musculoskeletal disorders (including muscle atrophy, weakness and pain, and neuromyopathy) and peripheral neuropathy also demonstrated a ROR indicative of safety signal, especially for CQ, although neuromyopathy as evidenced by abnormal nerve conduction and sensory changes is considered infrequent $(0.1 \%-1 \%)[16]$.

The strongest safety signals found in our analysis were for reporting cardiomyopathy, retinal disorder, corneal disorders, and arrhythmias. Interestingly, in all of them the ROR values were significantly higher for CQ, especially for cardiomyopathy (22.2 vs 3.2). The cardiac risk of CQ was further confirmed by the risk identified for arrhythmias (ROR 7.6). Cardiomyopathy and hearth rhythm problems are well recognized adverse events of CQ analogues, while both the FDA and EMA recently reminded health care professionals and patients of the known risks associated with both CQ and HCQ upon emerging reports of serious arrhythmias in patients with COVID-19 treated with CQ and HCQ, often in combination with azithromycin and other QT prolonging medicines [5,8]. Clinical data regarding the QTc-prolonging potential especially of chloroquine in COVID-19 patients is already published highlighting the need for ECG monitoring when prescribing chloroquine to COVID-19 patients [7].

In a double-blinded, randomized clinical trial from Brazil in patients with confirmed or suspected severe COVID-19, the safety of CQ diphosphate was assessed in dosages that were thought to be sufficient to exert antiviral effects. High dose CQ (600 mg BID) for 10 days was compared to lower-dose regimen, initially 450 $\mathrm{mg}$ BID on the first day, tapering to $450 \mathrm{mg}$ QD for 4 days and death was evaluated as the primary outcome [9]. Despite an intended sample size of 440 individuals, based on occurrence of serious adverse events, an independent data safety and monitoring board terminated the trial after the enrollment of only 81 individuals. By day 13 post-enrollment, $15.0 \%$ in the low-dose group had died, compared to $39.0 \%$ in the high-dose group, while prolongation of QTc interval was observed in $11.1 \%$ in the low-dose group and $18.9 \%$ in the high-dose group. However, potential confounders in this study included the co-administered drugs; all patients received azithromycin and the majority of them (86.8\% and $92.5 \%$ in the low- and high-dosage groups, respectively) oseltamivir, both of which are QTc prolongating drugs. The authors suggested that higher CQ dosage should not be recommended as a treatment for severe COVID-19, especially among patients receiving azithromycin and/or oseltamivir. Fihn and colleagues, in their response to the aforementioned study, suggest that prudent 
clinicians should discuss with patients and their families, when feasible, the potential risks of this drug and the uncertain benefits prior to initiation [17].

Central and peripheral Nervous system disorders including convulsions (ROR 2.6) and peripheral neuropathy (ROR 3.3) were also identified only for CQ and interestingly, a safety signal for psychosis (ROR 2.8) was also demonstrated. Many case reports and epidemiological studies have linked CQ analogues use, especially $\mathrm{CQ}$ and mefloquine, to an increased risk of developing neuropsychiatric adverse effects, such as depression or psychoses [18], while acute psychotic behavior has been associated with CQ decades ago [19]. A recently published disproportionality analysis of the FAERS focusing on the neuropsychiatric adverse events of CQ reported a significant, albeit marginal (aRORs between 1.3-2.1) association between the use of CQ and the reporting of amnesia, delirium, hallucinations, loss of consciousness and depression while a potential link between the use of CQ and an increased risk of suicide or psychosis was not detected. However, this analysis had some methodological limitations and included a limited time period of FAERS data (Q42012-Q42019) $[20]$.

One of our results raising concerns is the signal for reporting suicide and self-injury (ROR 4,19) for CQ. Death due to CQ overdosage has been reported even in its early years of use. In 1964 a report of 13 fatalities was published and suicide was the most likely cause of death in all cases. This 'milestone' publication highlighted the rapid time course of the overdose, since CQ is rapidly absorbed from the gastrointestinal tract, the onset of symptoms is fast, and death occurs in less than two hours [19]. Voluntary intoxication with CQ is a major medical and social problem especially in developing countries, where the drug is widely available. A study of 884 cases of self-poisoning in Mali revealed that the most common cases were suicide attempts and self-induced abortion and in the majority of them the drug used was CQ.[21] Since CQ is a common method of attempting suicide, there is much more toxicologic experience regarding to its toxicity. Based on studies, CQ is 2 to 3 times more toxic than HCQ [22]. The mortality rate in overdose studies in adults varies from $10 \%$ to $30 \%$ [23] and a $90 \%$ mortality rate was observed in a severe overdose case series [24]. In adults, the therapeutic dose is $10 \mathrm{mg} / \mathrm{kg}$, the toxic dose is $20 \mathrm{mg} / \mathrm{kg}$, and the lethal dose is $30 \mathrm{mg} / \mathrm{kg}$ [23]. CQ overdoses cause severe and rapid symptomatology, with symptoms frequently observed within 30 minutes. Death usually occurs within 1 to 3 hours after ingestion and the main cause of death is cardiac arrest. Cardiotoxicity occurs in greater than $50 \%$ of cases. Hypotension due to vasodilation and decreased cardiac output is frequently seen and occurs very quickly. Hypokalemia is identified in approximately $85 \%$ of CQ overdoses, and the decrease in potassium levels is associated with the severity of intoxication [25]. The pharmacokinetic parameters of CQ help explain the symptoms onset and dissipation. The drug is rapidly absorbed from the gastrointestinal tract, with a half-life of absorption of 30 minutes and a peak concentration achieved in 1.5 to 3 hours. These transiently high intravascular concentrations seen in the early distribution phase are associated with the cardiotoxic effects of CQ. As redistribution from this central compartment occurs, toxic effects diminish [24]. On the other hand $\mathrm{HC}$ overdoses are rarely reported in the literature and there is limited experience to characterize a HCQ overdose, although life-threatening symptoms can also occur rapidly following ingestion, most commonly within $1 \mathrm{~h}$, with very rapid progression to death within a few hours [26]. Little is known about HCQ kinetics in overdose except that plasma concentrations fit a 2-compartmental model, the Vd is $63 \mathrm{~L} / \mathrm{kg}$, and the half-life is 15.5 to 31 hours [22]. Due to the few number of HCQ overdose cases, there is no established lethal dose or toxic dose cut-off. One can assume that twice the CQ doses would be toxic for HCQ, however, the lethal plasma level of HCQ is not well established [26].

Our results suggesting a safety signal of CQ use and reporting suicide are in accordance with our finding in the multivariable logistic regression analysis that reports with the outcome death were more frequently associated with CQ use and co-reported suicide and self-injury. Death was also significantly associated with cardiomyopathy and cardiac arrhythmias, which are well-known complications of CQ/HCQ treatments in COVID-19 patients as well.

Our finding of a favorable safety profile of HCQ over CQ is not surprising. Differences in efficacy and toxicity between CQ and HCQ have also been reported in long-term users with rheumatoid diseases. HCQ is one half to two-thirds as effective as CQ, but half as toxic, in the treatment of rheumatic diseases. Additionally, while 
CQ exerts a number of severe side effects on fetal development, HCQ is strongly recommended in pregnant patients with an autoimmune disease, as it prevents the development of congenital heart block due to a potential inhibitory effect on type I interferon production. HCQ, rather than CQ, should be considered as a potential therapeutic solution for these patients, given its safety profile in pregnancy [27]. Nevertheless, the drug toxicity profile in COVID19 is currently unknown. It may prove to be dependent on both the patient population, the dose administered, and the concomitant drug therapies. Without knowing the effective drug concentrations, non-optimized dosing may expose patients to serious adverse effects. Thus some countries, including Australia, have already published guidance recommendations on off-label medicines use in the treatment and prophylaxis of COVID-19, recommending that HCQ use outside of a clinical trial should be avoided [28].

Our study has certain limitations. Pharmacovigilance databases like FAERS suffer from under-reporting, temporal patterns of reporting, notoriety of the adverse event and suboptimal quality of reports [11]. Further, disproportionality analysis is a statistical method that measures co-reporting of adverse events and drugs. It cannot remove the absolutely necessary step of a careful clinical causality assessment; thus, any identified association does not imply causation. Further, it is important to notice that 'death' may be reported as an adverse event (preferred term) or as an outcome (ie, as the final clinical event resulting from the adverse event). In the latter scenario, causality is even more challenging, because it is unfeasible to attribute the actual death to the drug, especially considering the complexity and diversity of patients. However, we deliberately focused our analyses on death as an outcome and performed a multivariable logistic regression analysis, because death is the most important outcome measure to validate the clinical profile of any drug in general. Finally, despite these limitations, the major strength of this study is the large number of reports, and the comprehensive list of adverse events. To our knowledge this is the first disproportionality analysis aiming to evaluate all FAERS data available on the safety profile of CQ and HCQ.

In conclusion, currently, there are very limited data to support the use of CQ and HCQ in the treatment or prevention of COVID-19. Clinical evidence is emerging, but efficacy results are inconclusive and a major concern regarding these drugs remains their safety profile. Our results confirm previously published evidence [29] and suggest that HCQ has a safer clinical profile compared to CQ, and thus could serve as a better therapeutic approach in COVID-19. However, until more real-world and RCTs' data are available, close supervision is strongly recommended.

\section{Acknowledgements: none}

Conflict of interest statement: none to declare

Funding information: none to declare

Author contribution: G. Papazisis: study design, literature search, data interpretation, manuscript drafting and editing; S. Siafis: study design, data collection, analysis, interpretation;D. Cepaityte: data collection and analysis, manuscript drafting; D. Giannis: literature search, manuscript editing; D. Tzachanis: literature search, manuscript editing;T. Egberts: data interpretation, manuscript editing

Data availability statement: Data that support the findings of the disproportionality analysis are not available, since the analysis was conducted within OpenVigil 2.1-MedDRA. The data and R code that support the findings of the analysis of reporting mortality are available from the corresponding author upon request

\section{References}

[1] Sanders JM, Monogue ML, Jodlowski TZ, Cutrell JB. Pharmacologic Treatments for Coronavirus Disease 2019 (COVID-19): A Review. JAMA - J Am Med Assoc 2020. https://doi.org/10.1001/jama.2020.6019.

[2] Gautret P, Lagier J-C, Parola P, Hoang VT, Meddeb L, Mailhe M, et al. Hydroxychloroquine and azithromycin as a treatment of COVID-19: results of an open-label non-randomized clinical trial. Int J Antimicrob Agents 2020:105949. https://doi.org/10.1016/j.ijantimicag.2020.105949. 
[3] International Society of Antimicrobial Chemotherapy. Official statement. Assoc Med J 1854;s3-2:68-70. https://doi.org/10.1136/bmj.s3-2.55.68.

[4] Gao J, Tian Z, Yang X. Breakthrough: Chloroquine phosphate has shown apparent efficacy in treatment of COVID-19 associated pneumonia in clinical studies. Biosci Trends 2020;14. https://doi.org/10.5582/BST.2020.01047.

[5] U.S. Food and Drug Administration. Emergency Use Authorization (EUA) - CytoSorb. n.d. https://www.fda.gov/media/136534/download (accessed April 5, 2020).

[6] Inciardi RM, Lupi L, Zaccone G, Italia L, Raffo M, Tomasoni D, et al. Cardiac Involvement in a Patient with Coronavirus Disease 2019 (COVID-19). JAMA Cardiol 2020. https://doi.org/10.1001/jamacardio.2020.1096.

[7] van den Broek MPH, Möhlmann JE, Abeln BGS, Liebregts M, van Dijk VF, van de Garde EMW. Chloroquine-induced QTc prolongation in COVID-19 patients. Netherlands Hear J 2020:1-4. https://doi.org/10.1007/s12471-020-01429-7.

[8] The European Medicines Agency - EMA. COVID-19: reminder of risk of serious side effects with chloroquine and hydroxychloroquine 2020;31:1-2. https://doi.org/10.1101/2020.04.07.20056424.

[9] Borba MGS, Val FFA, Sampaio VS, Alexandre MAA, Melo GC, Brito M, et al. Effect of High vs Low Doses of Chloroquine Diphosphate as Adjunctive Therapy for Patients Hospitalized With Severe Acute Respiratory Syndrome Coronavirus 2 (SARS-CoV-2) Infection. JAMA Netw Open 2020. https://doi.org/10.1001/jamanetworkopen.2020.8857.

[10] U.S. Food \& Drug Administration. FDA Drug Safety Communication: FDA cautions against use of hydroxychloroquine or chloroquine for COVID-19 outside of the hospital setting or a clinical trial due to risk of heart rhythm problems 2020. www.clinicaltrials.gov (accessed May 3, 2020).

[11] Sakaeda T, Tamon A, Kadoyama K, Okuno Y. Data mining of the public version of the FDA adverse event reporting system. Int J Med Sci 2013;10:796-803. https://doi.org/10.7150/ijms.6048.

[12] Van Puijenbroek EP, Bate A, Leufkens HGM, Lindquist M, Orre R, Egberts ACG. A comparison of measures of disproportionality for signal detection is spontaneous reporting systems for adverse drug reactions. Pharmacoepidemiol Drug Saf 2002;11:3-10. https://doi.org/10.1002/pds.668.

[13] Al-Bari AA. Chloroquine analogues in drug discovery: New directions of uses, mechanisms of actions and toxic manifestations from malaria to multifarious diseases. J Antimicrob Chemother 2014;70:1608-21. https://doi.org/10.1093/jac/dkv018.

[14] Freund NT, Roitburd-Berman A, Sui J, Marasco WA, Gershoni JM. Reconstitution of the receptor-binding motif of the SARS coronavirus. Protein Eng Des Sel 2015;28:567-75. https://doi.org/10.1093/protein/gzv052.

[15] Jorge A, Ung C, Young LH, Melles RB, Choi HK. Hydroxychloroquine retinopathy implications of research advances for rheumatology care. Nat Rev Rheumatol 2018;14:693-703. https://doi.org/10.1038/s41584-018-0111-8.

[16] Rossi S. Australian Medicines Handbook. Encycl Clin Pharm 2002:75-8. https://doi.org/10.3109/9780824706081.019.

[17] Fihn SD, Perencevich E, Bradley SM. Caution Needed on the Use of Chloroquine and Hydroxychloroquine for Coronavirus Disease 2019. JAMA Netw Open 2020;3:e209035. https://doi.org/10.1001/jamanetworkopen.2020.9035.

[18] Meier CR, Wilcock K, Jick SS. The Risk of Severe Depression, Psychosis or Panic Attacks with Prophylactic Antimalarials. Drug Saf 2004;27:203-13. https://doi.org/10.2165/00002018-200427030-00005. 
[19] Kiel FW. Chloroquine Suicide. JAMA J Am Med Assoc 1964;190:398-400. https://doi.org/10.1001/jama.1964.03070170139030.

[20] Sato K, Mano T, Iwata A, Toda T. Neuropsychiatric adverse events of chloroquine: a real-world pharmacovigilance study using the FDA Adverse Event Reporting System (FAERS) database. Biosci Trends 2020. https://doi.org/10.5582/bst.2020.03082.

[21] Diallo T, Hami H, Maiga A, Coulibaly B, Maiga D, Mokhtari A, et al. [Epidemiology and risk factors for voluntary intoxication in Mali]. Sante Publique 2013;25:359-66. https://doi.org/10.3917/spub.253.0359.

[22] Jordan P, Brookes JG, Nikolic G, Le Couteur DG. Hydroxychloroquine overdose: Toxicokinetics and management. J Toxicol - Clin Toxicol 1999;37:861-4. https://doi.org/10.1081/CLT-100102466.

[23] Jaeger A, Sauder P, Kopferschmitt J, Flesch F. Clinical Features and Management of Poisoning due to Antimalarial Drugs. Med Toxicol Adverse Drug Exp 1987;2:242-73. https://doi.org/10.1007/BF03259868.

[24] Riou B, Barriot P, Rimailho A, Baud FJ. Treatment of Severe Chloroquine Poisoning. N Engl J Med 1988;318:1-6. https://doi.org/10.1056/NEJM198801073180101.

[25] Clemessy JL, Borron SW, Baud FJ, Favier C, Hantson PE, Vicaut E. Hypokalaemia related to acute chloroquine ingestion. Lancet 1995;346:877-80. https://doi.org/10.1016/S0140-6736(95)92711-5.

[26] Marquardt K, Albertson TE. Treatment of hydroxychloroquine overdose. Am J Emerg Med 2001;19:4204. https://doi.org/10.1053/ajem.2001.25774.

[27] Izmirly PM, Costedoat-Chalumeau N, Pisoni CN, Khamashta MA, Kim MY, Saxena A, et al. Maternal use of hydroxychloroquine is associated with a reduced risk of recurrent anti-SSA/roantibody - Associated cardiac manifestations of neonatal lupus. Circulation 2012;126:76-82. https://doi.org/10.1161/CIRCULATIONAHA.111.089268.

[28] Kalil AC. Treating COVID-19 - Off-Label Drug Use, Compassionate Use, and Randomized Clinical Trials during Pandemics. JAMA - J Am Med Assoc 2020. https://doi.org/10.1001/jama.2020.4742.

[29] Zhou D, Dai SM, Tong Q. COVID-19: a recommendation to examine the effect of hydroxychloroquine in preventing infection and progression. J Antimicrob Chemother 2020. https://doi.org/10.1093/jac/dkaa114.

Table 1. Disproportionality analysis of chloroquine and hydroxychloroquine

\begin{tabular}{llllll}
\hline & Chloroquine & Chloroquine & Chloroquine & Chloroquine & Hydroxychloroqui \\
\hline & $\mathbf{n}$ & ROR & LB_ROR & UP_ROR & $\mathbf{n}$ \\
Cardiomyopathy & 53 & $\mathbf{2 2 . 2}$ & $\mathbf{1 6 . 8}$ & $\mathbf{2 9 . 3}$ & 222 \\
Retinal disorder & 53 & $\mathbf{1 2 . 6}$ & $\mathbf{9 . 6}$ & $\mathbf{1 6 . 7}$ & 493 \\
Corneal disorders & 7 & $\mathbf{8 . 7}$ & $\mathbf{4 . 1}$ & $\mathbf{1 8 . 3}$ & 39 \\
Cardiac Arrythmias & 98 & $\mathbf{7 . 6}$ & $\mathbf{6 . 1}$ & $\mathbf{9 . 3}$ & 465 \\
Severe cutaneous reactions & 15 & $\mathbf{2 . 9}$ & $\mathbf{1 . 8}$ & $\mathbf{4 . 9}$ & 343 \\
Decreased appetite & 17 & $\mathbf{1 . 9}$ & $\mathbf{1 . 2}$ & $\mathbf{3 . 1}$ & 254 \\
Psychotic disorders & 22 & $\mathbf{2 . 8}$ & $\mathbf{1 . 8}$ & $\mathbf{4 . 2}$ & 82 \\
Convulsions & 33 & $\mathbf{2 . 6}$ & $\mathbf{1 . 8}$ & $\mathbf{3 . 7}$ & 200 \\
Headache & 45 & $\mathbf{1 . 7}$ & $\mathbf{1 . 3}$ & $\mathbf{2 . 3}$ & 1071 \\
Hypoglycaemia & 4 & 0.8 & 0.3 & 2.1 & 94 \\
Extrapyramidal symptoms & 5 & 0.6 & 0.2 & 1.4 & 64 \\
Hearing and vestibular disorders & 20 & $\mathbf{2 . 4}$ & $\mathbf{1 . 5}$ & $\mathbf{3 . 7}$ & 302 \\
Peripheral neuropathy & 21 & $\mathbf{3 . 3}$ & $\mathbf{2 . 1}$ & $\mathbf{5 . 0}$ & 186 \\
Suicide and self-injury & 28 & $\mathbf{4 . 2}$ & $\mathbf{3 . 3}$ & $\mathbf{5 . 4}$ & 540 \\
Hepatic disorders & 75 & $\mathbf{3 . 1}$ & $\mathbf{2 . 5}$ & $\mathbf{4 . 0}$ & 1193 \\
Depression & 20 & 1.2 & 0.8 & 1.9 & 285
\end{tabular}




\begin{tabular}{llllll}
\hline & Chloroquine & Chloroquine & Chloroquine & Chloroquine & Hydroxychloroqui \\
\hline Gastrointestinal symptoms & 96 & 1.2 & 0.9 & 1.4 & 3027 \\
Hypersensitivity & 85 & 1.2 & 1.0 & 1.5 & 3587 \\
Haematopoietic cytopenia & 45 & $\mathbf{2 . 0}$ & $\mathbf{1 . 5}$ & $\mathbf{2 . 7}$ & 899 \\
Musculoskeletal disorders & 32 & $\mathbf{2 . 0}$ & $\mathbf{1 . 4}$ & $\mathbf{2 . 9}$ & 659 \\
Lactose intolerance & 0 & 0.0 & 0.0 & 0.0 & 1 \\
\hline
\end{tabular}

Table 2. Multivariable logistic regression for reporting outcome death of chloroquine and hydroxychloroquine

\begin{tabular}{lllll}
\hline factor & OR & LB_OR & UP_OR & p-value \\
\hline Suicide and self-injury & $\mathbf{8 . 3 7}$ & $\mathbf{6}$ & $\mathbf{1 1 . 7}$ & $\mathbf{0}$ \\
Cardiomyopathy & $\mathbf{3 . 0 5}$ & $\mathbf{2 . 1 3}$ & $\mathbf{4 . 4}$ & $\mathbf{0}$ \\
Cardiac Arrhythmias & $\mathbf{1 . 6 8}$ & $\mathbf{1 . 2 3}$ & $\mathbf{2 . 3}$ & $\mathbf{0}$ \\
Decreased appetite & $\mathbf{2 . 3}$ & $\mathbf{1 . 2}$ & $\mathbf{4 . 4}$ & $\mathbf{0 . 0 1}$ \\
Gender (Male) & $\mathbf{1 . 4 3}$ & $\mathbf{1 . 1 9}$ & $\mathbf{1 . 7}$ & $\mathbf{0}$ \\
Reporting country (US) & $\mathbf{1 . 2 6}$ & $\mathbf{1 . 0 5}$ & $\mathbf{1 . 5}$ & $\mathbf{0 . 0 1}$ \\
Age in report & $\mathbf{1 . 0 2}$ & $\mathbf{1 . 0 1}$ & $\mathbf{1 . 0 2}$ & $\mathbf{0}$ \\
Hypoglycaemia & 4.85 & 0.96 & 24.5 & 0.06 \\
Date of the report & $\mathbf{0 . 9 5}$ & $\mathbf{0 . 9 3}$ & $\mathbf{0 . 9 8}$ & $\mathbf{0}$ \\
Hepatic disorders & 1.06 & 0.78 & 1.4 & 0.72 \\
Severe cutaneous reactions & 1.31 & 0.72 & 2.4 & 0.37 \\
Systematic lupus erythematosus & 0.88 & 0.69 & 1.1 & 0.27 \\
Haematopoietic cytopenia & 0.95 & 0.67 & 1.3 & 0.76 \\
Hypersensitivity & $\mathbf{0 . 6 7}$ & $\mathbf{0 . 5}$ & $\mathbf{0 . 9}$ & $\mathbf{0 . 0 1}$ \\
Gastrointestinal symptoms & $\mathbf{0 . 6 6}$ & $\mathbf{0 . 4 9}$ & $\mathbf{0 . 9}$ & $\mathbf{0 . 0 1}$ \\
Musculoskeletal disorders & 0.78 & 0.47 & 1.3 & 0.34 \\
Drug (HCQ) & $\mathbf{0 . 6}$ & $\mathbf{0 . 4 4}$ & $\mathbf{0 . 8}$ & $\mathbf{0}$ \\
Drug (CQ) & $\mathbf{1 . 7}$ & $\mathbf{1 . 3}$ & $\mathbf{2 . 3}$ & $\mathbf{0}$ \\
Arthritis & $\mathbf{0 . 4 5}$ & $\mathbf{0 . 3 8}$ & $\mathbf{0 . 5}$ & $\mathbf{0}$ \\
Malaria & 0.74 & 0.31 & 1.8 & 0.5 \\
Corneal disorders & 1.37 & 0.3 & 6.2 & 0.68 \\
Headache & $\mathbf{0 . 4 2}$ & $\mathbf{0 . 2 2}$ & $\mathbf{0 . 8}$ & $\mathbf{0 . 0 1}$ \\
Depression & $\mathbf{0 . 4 2}$ & $\mathbf{0 . 1 9}$ & $\mathbf{0 . 9}$ & $\mathbf{0 . 0 2}$ \\
Peripheral neuropathy & 0.37 & 0.13 & 1 & 0.06 \\
Convulsions & 0.44 & 0.09 & 2.2 & 0.31 \\
Psychotic disorders & $\mathbf{0 . 0 7}$ & $\mathbf{0 . 0 1}$ & $\mathbf{0 . 5}$ & $\mathbf{0 . 0 1}$ \\
Retinal disorders & $\mathbf{0 . 0 3}$ & $\mathbf{0}$ & $\mathbf{0 . 2}$ & $\mathbf{0}$ \\
Hearing and vestibular disorders & 0 & 0 & INF & 0.94 \\
Extrapyramidal symptoms & 0 & 0 & INF & 0.98 \\
\hline
\end{tabular}

Figure Legends

Figure 1: Disproportionality analysis of chloroquine and hydroxychloroquine

Figure 2: Multivariable logistic regression for reporting outcome death of chloroquine and hydroxychloroquine 


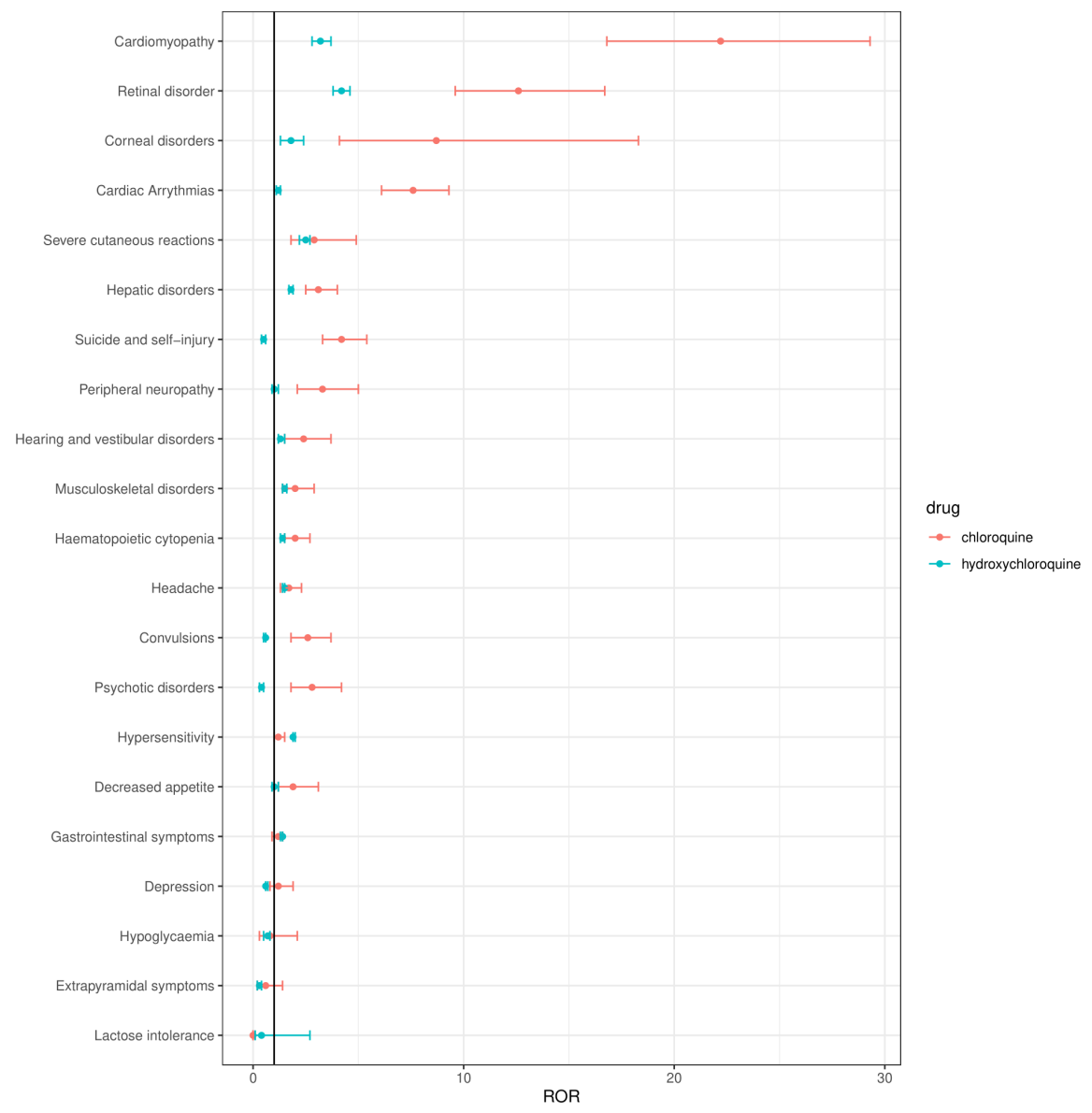




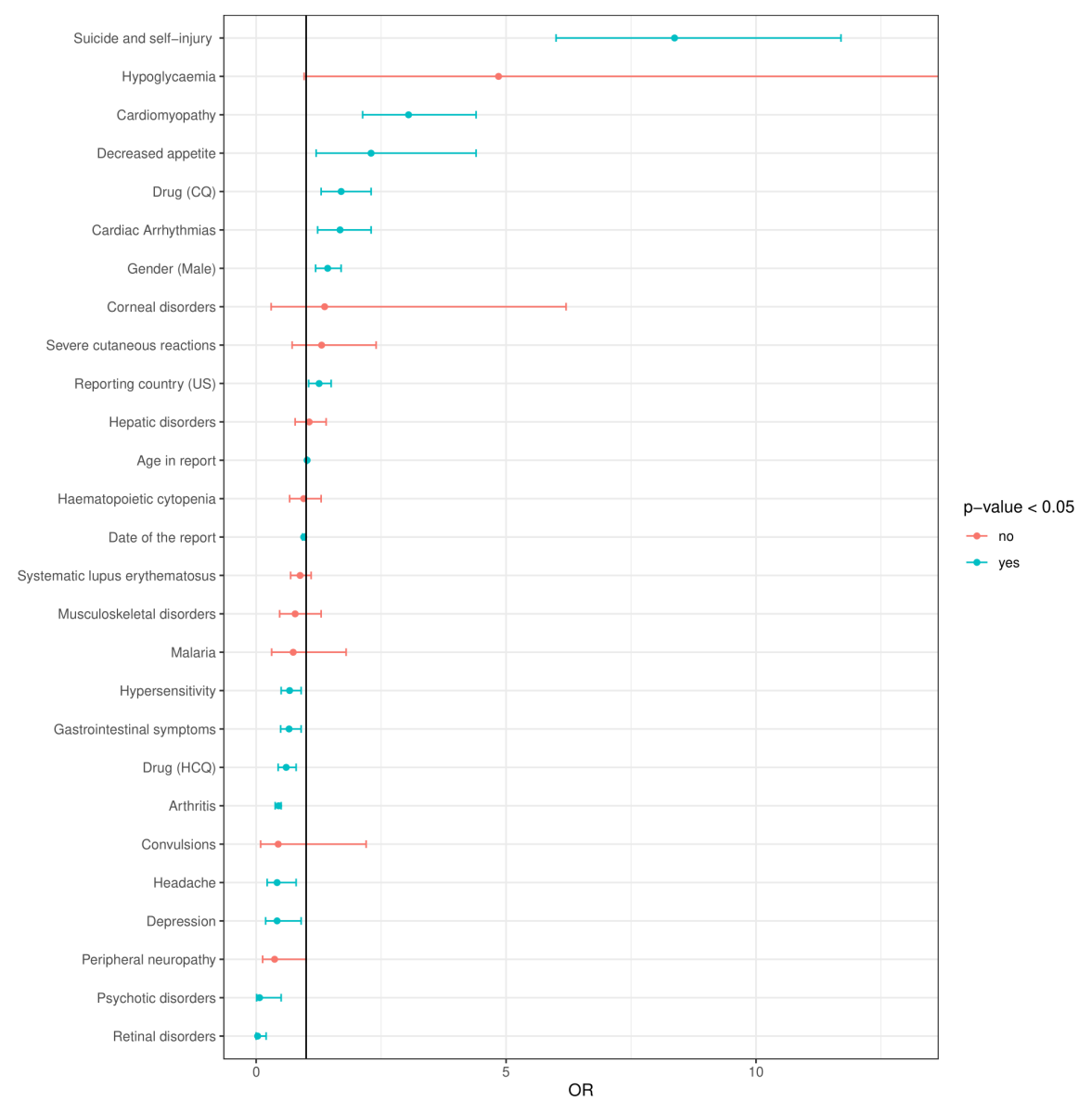

